\title{
Relationships between electron density, height and sub-peak ionospheric thickness in the night equatorial ionosphere
}

\author{
K. J. W. Lynn ${ }^{1}$, T. J. Harris ${ }^{2}$, and M. Sjarifudin ${ }^{3}$ \\ ${ }^{1}$ Ionospheric Systems Research, Noosaville, Australia \\ ${ }^{2}$ Defence Science \& Technology Organisation, P.O. Box 1500, Edinburgh, Australia \\ ${ }^{3}$ National Institute for Aeronautics and Space (LAPAN), Bandung, Indonesia \\ Received: 10 August 2005 - Revised: 19 December 2005 - Accepted: 9 January 2006 - Published: 3 July 2006 \\ Part of Special Issue "The 11th International Symposium on Equatorial Aeronomy (ISEA-11), Taipei, May 2005”
}

\begin{abstract}
The development and decay of the southern equatorial anomaly night-time peak in electron density as seen at a number of ionosonde reflection points extending from New Guinea and Indonesia into northern Australia was examined in terms of the characteristic rise and fall in height associated with the sunset ionisation-drift vortex at the magnetic equator. The observations relate to measurements made in November 1997. Following sunset, the ionospheric profile was observed to narrow as the maximum electron density increased during a fall in height that took the peak of the layer at Vanimo and Sumedang down to some $240 \mathrm{~km}$. The fall was followed by a strong rise in which the electron density sub-peak profile expanded from a slab width (as given by POLAN) of $20 \mathrm{~km}$ to over $100 \mathrm{~km}$ with no corresponding change in peak electron density. The post-sunset equatorial fall in height and associated changes in profile density and thickness continued to be seen with diminishing amplitude and increasing local time delay in moving from the anomaly peak at Vanimo to the southernmost site of observation at Townsville. Secondary events on a lesser scale sometimes occurred later in the night and may provide evidence of the multiple vortices suggested by Kudeki and Bhattacharyya (1999). Doppler measurements of vertical velocity as seen at Sumedang in Java are compared with the observed changes in electron density profile in the post-sunset period. The normal post-sunset variation in ionospheric parameters was disrupted on the night of 7 November, the night before a negative ionospheric storm was observed.
\end{abstract}

Keywords. Ionosphere (Equatorial ionosphere, Ionospheric disturbances, Electric fields and currents)

Correspondence to: K. J. W.Lynn

(kenjwlynn@bigpond.com)

\section{Introduction}

An upward vertical drift of ionisation characteristically occurs at equatorial latitudes around sunset at F2 heights in the ionosphere. A post-sunset reversal from upward to downward vertical drift follows later in the night (Fejer, 1991; Fejer et al., 1995). There is an accompanying lift and fall in F2 layer height associated with the changing drift pattern. At Southeast Asian longitudes, the phenomenon is usually most evident near the equinoxes. More recently, incoherent scatter radar measurements at Jicamarca (Kudeki and Bhattacharyya, 1999) and satellite measurements (Eccles et al., 1999) have produced a more detailed picture of changing plasma drifts with height through the sunset period and into the night. These measurements show that the upward and downward drifts at F2 heights pre-midnight are part of a larger vortex pattern centered at some $250 \mathrm{~km}$ in height and around 20 LT in time. As originally suggested by Tsunoda, 1981, the base of the vortex is formed by a westward drift in the $\mathrm{E}$ region which corresponds to an eastward drift at F2 heights. The existence of a vortex in $\boldsymbol{E} \times \boldsymbol{B}$ drift implies a divergent field structure with $E$ field vectors pointing to a negatively charged core region (Kudeki and Bhattacharyya, 1999).

While the vertical component of drift associated with sunset has long been known to be the result of $\boldsymbol{E} \times \boldsymbol{B}$ forces, a full theoretical explanation has been more difficult to determine. The upward drift which produces the daytime equatorial anomaly is produced by eastward electric fields generated in the $\mathrm{E}$ region. The resurgence of the equatorial anomaly at night associated with an increase in vertical drift around sunset and its subsequent reversal is a more complex phenomenon involving both $\mathrm{E}$ and $\mathrm{F}$ region dynamos. Theories to explain the observed vortex motion are discussed by

Published by Copernicus GmbH on behalf of the European Geosciences Union. 


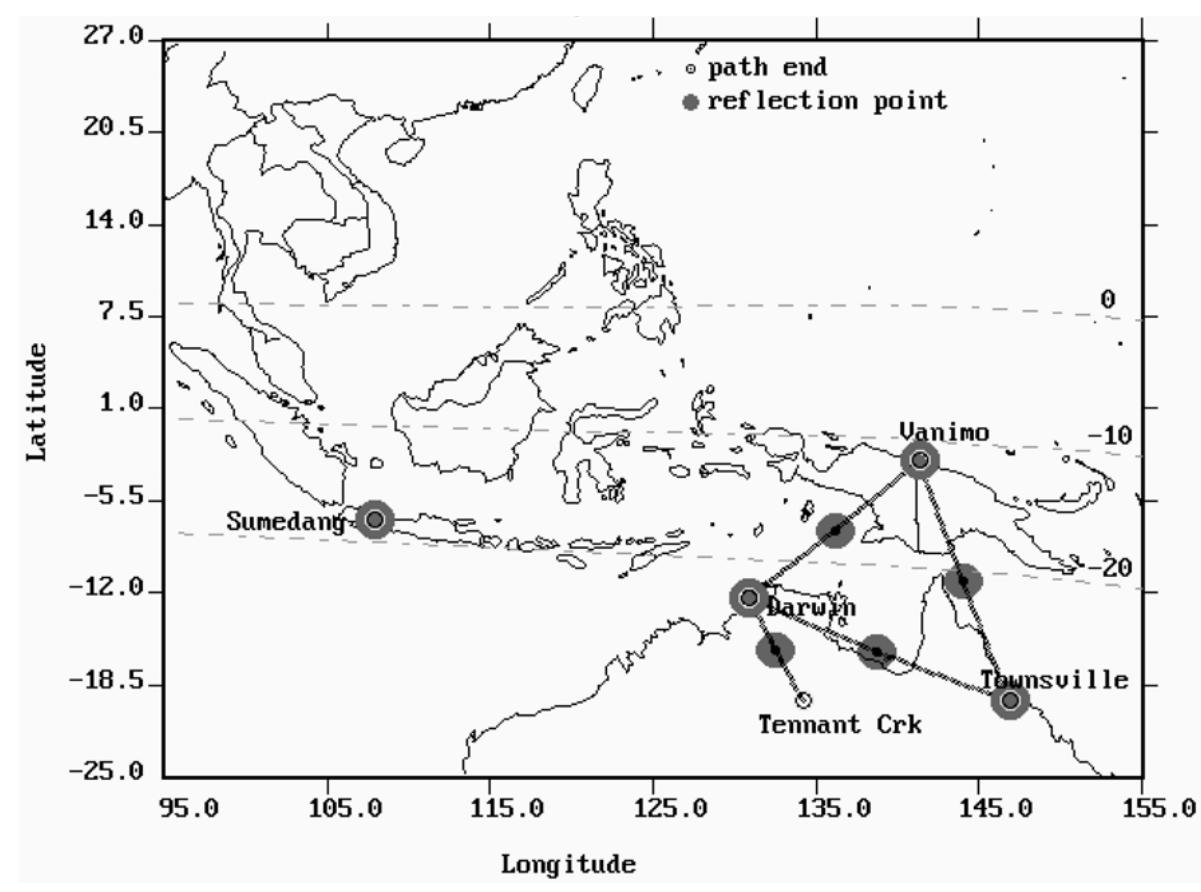

Fig. 1. Location of ionosondes and reflection points.

Eccles (1998). An empirical global equatorial model of $F$ region vertical drift has been prepared by Scherliess and Fejer (1999) based on radar and satellite measurements. The dependence of post-sunset vertical drift magnitude on solar flux is discussed by Whalen (2004).

In daytime, ionisation lifted by $\boldsymbol{E} \times \boldsymbol{B}$ forces at the magnetic equator diffuses down the field lines where it accumulates forming the equatorial anomaly which consists of twin peaks of electron density in the F2 region symmetrically disposed around the magnetic equator. Although the magnitude of the night-time equatorial anomaly still depends on the strength of the post-sunset vertical drift (Whalen, 2004), the build-up of the anomaly itself occurs mainly during the post-sunset reversal to downward drift associated with the eastern side of the sunset vortex. Balan and Bailey, 1995 refer to this as the reverse plasma fountain and illustrate the subsequent development of the night-time anomaly as given by the Sheffield University plasmasphere-ionosphere model.

The post-sunset period is also marked by the development of equatorial bubbles produced by the Rayleigh-Taylor instability. These bubbles are often seen as Equatorial Spread $\mathrm{F}$ (EQSF). The occurrence of such bubbles reaches a maximum when the sunset terminator aligns with the Earth's magnetic field lines (Tsunoda, 1985). This alignment occurs near the equinoxes where the Earth's magnetic field declination is small as at Southeast Asian longitudes. Outstanding problems in the relationship between the post-sunset height rise and fall and the occurrence of equatorial spread $\mathrm{F}$ are discussed by Abdu (2001).
This paper examines the relationships between $f o \mathrm{~F} 2$, virtual and true height, sub-peak slab thickness and Doppler shift in reflected radio signals in the post sunset ionosphere as seen by ionosondes near the peak of the night equatorial anomaly. The ionosondes were situated at various sites in and to the north of Australia. These latitudes cover an area where variations in observed ionospheric characteristics at any given time are largely associated with the development and magnitude of the day and night equatorial anomalies which in turn are driven by changes in ionisation drift at the magnetic equator.

\section{Observing sites}

Sites and paths of vertical and oblique ionosondes discussed in this paper are shown in Fig. 1. The vertical ionosondes at Vanimo and Townsville were operated by the Australian Ionospheric Prediction Service (IPS). Oblique ionosondes were operated by the Australian Defence Science and Technology Organisation (DSTO) between a transmitter at Vanimo and receivers at Darwin and Townsville. All other oblique path ionograms were obtained with DSTO equipment. A KEL IPS71 ionosonde at Sumedang (also called Tanjungsari) in Java was operated by the Indonesian National Institute for Aeronautics and Space (LAPAN). This ionosonde has the additional unique feature of being able to record high resolution Doppler ionograms. 

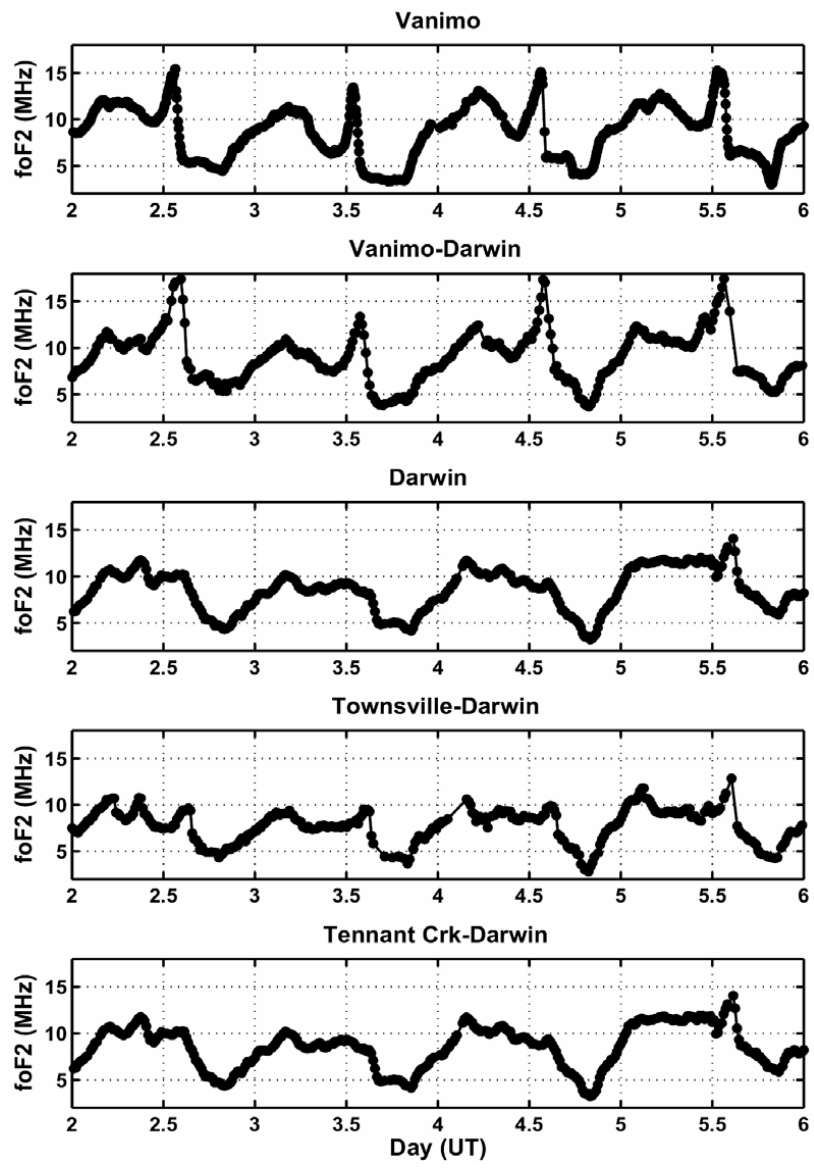

Fig. 2. Diurnal variations in $f o \mathrm{~F} 2$ at ionospheric reflection points of increasing latitude observed over the period 2-6 November 1997.

\section{Latitudinal variation in $f o \mathbf{F} 2$}

In early November, 1997, unusually large surges in postsunset values of $f o \mathrm{~F} 2$ were observed at Vanimo. Data from other sites were sought in order to obtain some idea of the latitudinal and longitudinal extent of these extreme variations. Ionograms over the oblique paths were converted to equivalent verticals from which $f o \mathrm{~F} 2$ could be obtained.

As seen in Fig. 2, post-sunset surges in $f o \mathrm{~F} 2$ were strongly present at Vanimo and over the oblique path Vanimo-Darwin. The peak values in $f o \mathrm{~F} 2$ at such times greatly exceeded $f o \mathrm{~F} 2$ values observed at any other time of day. Such surges, with diminishing magnitude, continued to be seen at reflection points further south. The surge on the night of 5 November is particularly notable for being clearly visible at all sites. While post-sunset surges in $f o \mathrm{~F} 2$ were also present at Sumedang during this time, variations in the magnitude of the post-sunset surges at Sumedang were uncorrelated with those at the eastern sites.

It should be pointed out that while the post-sunset surges in $f o \mathrm{~F} 2$ seen at Vanimo and discussed here represent extreme examples, surges of lesser magnitude are normally present at
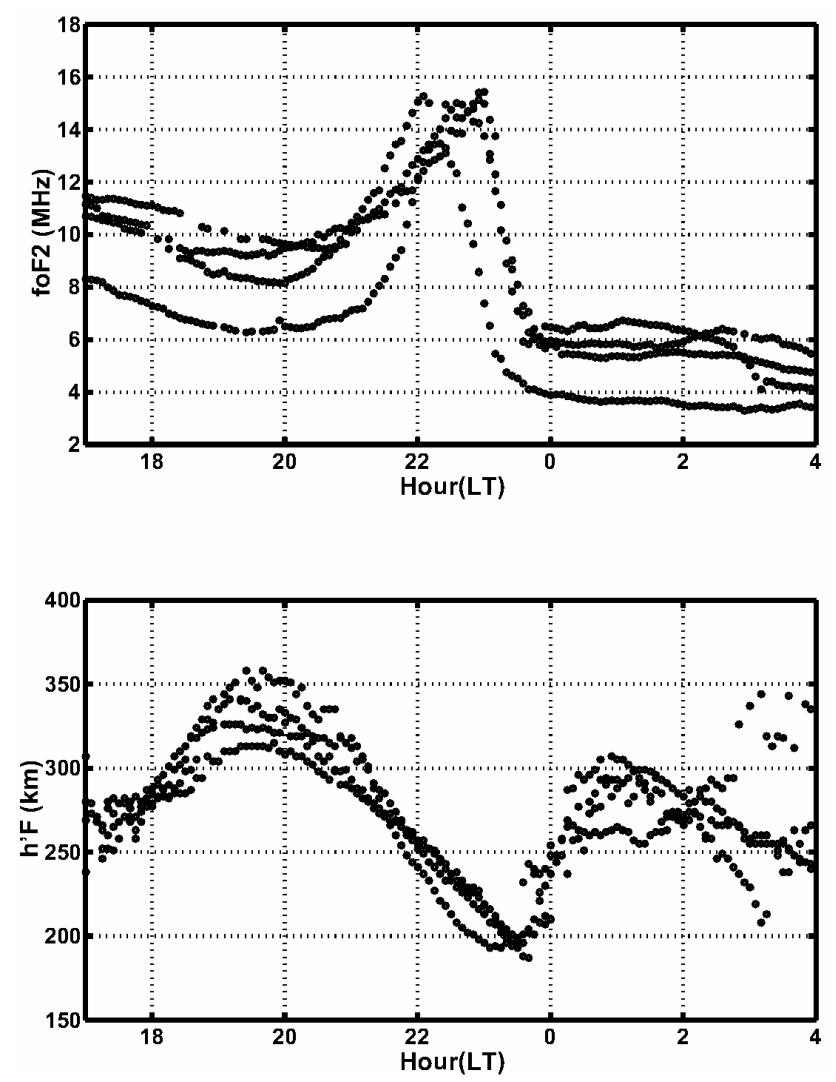

Fig. 3. Superimposed variations in $f o \mathrm{~F} 2$ (upper) and $\mathrm{h}^{\prime} \mathrm{F}$ (lower) observed at Vanimo over the period 1-4 November 1997.

magnetic latitudes corresponding to Vanimo and Sumedang. The post-sunset surge represents the time-dependent build up and decay of the night-time southern equatorial anomaly peak at these latitudes. The period discussed here (18 November 1997) was one of relatively low sunspot number (39) rising from the sunspot minimum in 1995-1996.

\section{Relations between electron density, height and iono- spheric thickness}

In Fig. 3, diurnal values of $f_{o} \mathrm{~F} 2$ and ionospheric base height $\mathrm{h}^{\prime} \mathrm{F}$ at Vanimo are superimposed to demonstrate the time and magnitude consistency of the post-sunset surges in $f o \mathrm{~F} 2$ and the associated height variations. The pre-reversal increase in ionospheric height reached its maximum at approximately 19:30 LT. From there the ionosphere descended continuously for the next four hours before a relatively abrupt recovery starting at approximately 23:30 LT. The maximum electron density of the ionosphere as measured by $f o \mathrm{~F} 2$ builds up as the ionosphere falls reaching a peak around 22-23 LT before decreasing to steady night values by the time the fall in ionospheric height has finished (just prior to local midnight). 

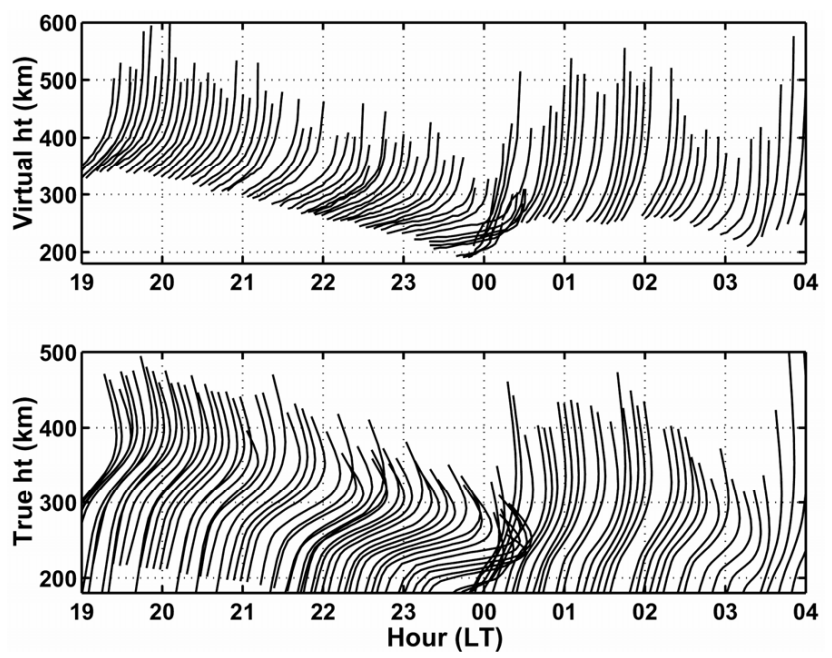

Fig. 4. Ionograms (upper) and the derived true height profiles given by POLAN (lower) measured on the night of 4 November 1997 and plotted as a function of time.

The program POLAN (Titheridge, 1988) was used to convert virtual height to true height. Virtual and true height electron density profiles (expressed as a plasma frequency) are shown in Fig. 4 as obtained at Vanimo on the night of 4 November. Here a sharpening of the ionospheric profile is seen to be associated with an increase in maximum electron density as the ionosphere falls. An abrupt recovery to a broader profile with an increase in peak height occurs at the end of the fall in height. This post-reversal recovery in peak height and profile thickness was not associated with any particular change in $f o \mathrm{~F} 2$.

There is a new fall in ionospheric height with an associated sharpening of the profile and subsequent recovery in the period 01-03 LT which will be discussed further in Sect. 7. While much weaker than the previous surge, this lesser event exhibits the same characteristics in all parameters and is typical of such events occasionally observed to occur in the postmidnight period.

A selection of maximum ionospheric electron density values (measured as a critical frequency) and base $\left(\mathrm{h}^{\prime} \mathrm{f}\right)$ or true height $(h m \mathrm{~F} 2)$ values is given in Figs. 5a-e as a function of time. Figures 5a, d and, e show the calculated results of POLAN for both the magnitude and variation in true height of the maximum electron density value as well as the equivalent slab thickness $\mathrm{W}$ of the sub-peak ionosphere. Here the slab thickness $W$ uses the definition given by POLAN as the integrated sub-peak electron density divided by the peak electron density. As such it is a measure of the profile width between the base of the F2 layer and the layer peak.

As seen at Vanimo in Fig. 5a (4 November 1997), The fall in ionospheric peak height $(h m \mathrm{~F} 2)$ begins at 19:00 LT and continues over a 3.8-h period at a rate increasing from some $7 \mathrm{~m} / \mathrm{s}$ to $14 \mathrm{~m} / \mathrm{s}(12 \mathrm{~m} / \mathrm{s}$ average $)$. An abrupt recovery to a broader profile with an increase in peak height commences at 23:30 LT. This recovery lasted some $25 \mathrm{~min}$ with an average rise velocity of $67 \mathrm{~m} / \mathrm{s}$. The fall in height of the peak F2 electron density from $400 \mathrm{~km}$ to $240 \mathrm{~km}$ is accompanied by a reduction in sub-peak slab thickness $W$ by a factor of three. A similar variation was seen at Sumedang on 1 November 1997, although here the maximum increase in electron density is seen to be less. Both Vanimo and Sumedang lie near the peak of the southern equatorial night-time anomaly. The fall in peak layer height at both Vanimo and Sumedang ended at a height around $240 \mathrm{~km}$. At this time, the difference between base and peak F2 layer height at Vanimo was about $50 \mathrm{~km}$. The maximum in $f o \mathrm{~F} 2$ values occurred as the layer peak fell below some $250-270 \mathrm{~km}$. The subsequent reduction in $f o \mathrm{~F} 2$ can be attributed to the rapid increase in recombination rate at these low heights. Thus the exact time at which the $f o \mathrm{~F} 2$ values peak can be expected to occur may depend on the rapidity with which the height falls. There was a lesser though similar variation of all three parameters with a shift in occurrence to later in the night on 4 November 1997 at the southernmost site of Townsville (Fig. 5d).

True height profiles for the oblique path from Vanimo to Townsville and at Darwin were not available but variations in maximum frequency and minimum time delay (Fig. 5b, 4 November 1997) and base F2 virtual height $h^{\prime} F$ (Fig. 5c, 5 November 1997) are included to show that there was a similar relationship between falling height and surging maximum frequency at these ionospheric reflection points.

\section{$5 \quad$ Latitudinal time variation}

A time shift is evident between Vanimo and Townsville surge events on 4 November 1997 shown in Fig. 5. Verification of this feature as a consistent result was obtained by superimposing a number of base ionospheric height variations $\left(\mathrm{h}^{\prime} \mathrm{F}\right)$ measured at Vanimo, Darwin and Townsville over the same nights with results shown in Fig. 6.

Peak ionospheric base height at Vanimo is seen to occur within two hours of ground-sunset. The subsequent fall in height ends just before local midnight. At Darwin, the peak height is reached some three to four hours after local groundsunset and the fall in height finishes just after local midnight. At the southernmost site of Townsville, the peak height is reached slightly later than at Darwin and the subsequent fall ends between one and two hours after local midnight.

\section{Doppler observations at Sumedang}

The Kel IPS71 ionosonde at Sumedang recorded Doppler ionograms at a 15-min rate. At each sweep frequency a Doppler Spectrum was taken and the Doppler frequency with the maximum amplitude value was recorded. These Doppler 
(a) 4 Nov. 1997 VANIMO
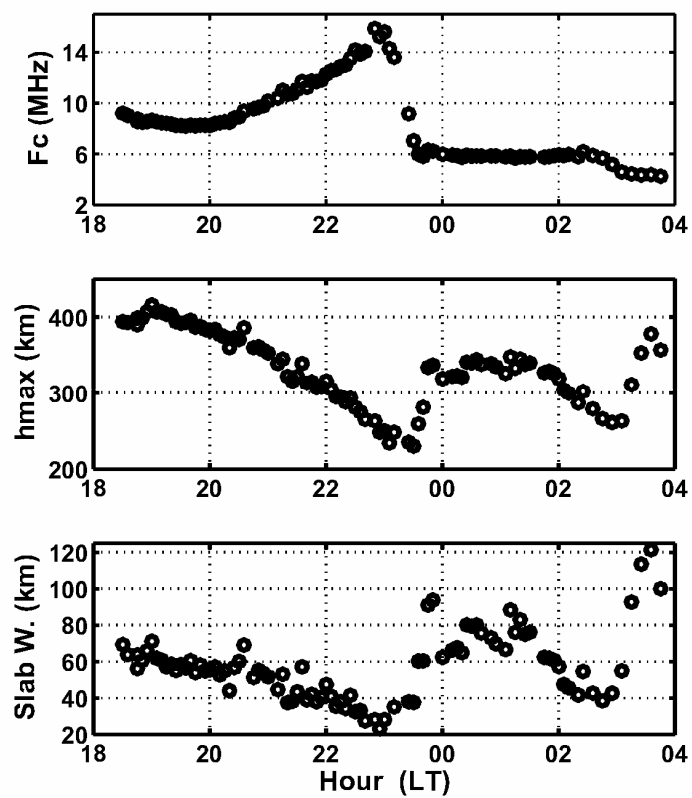

(d) 4 Nov.1997 Townsville
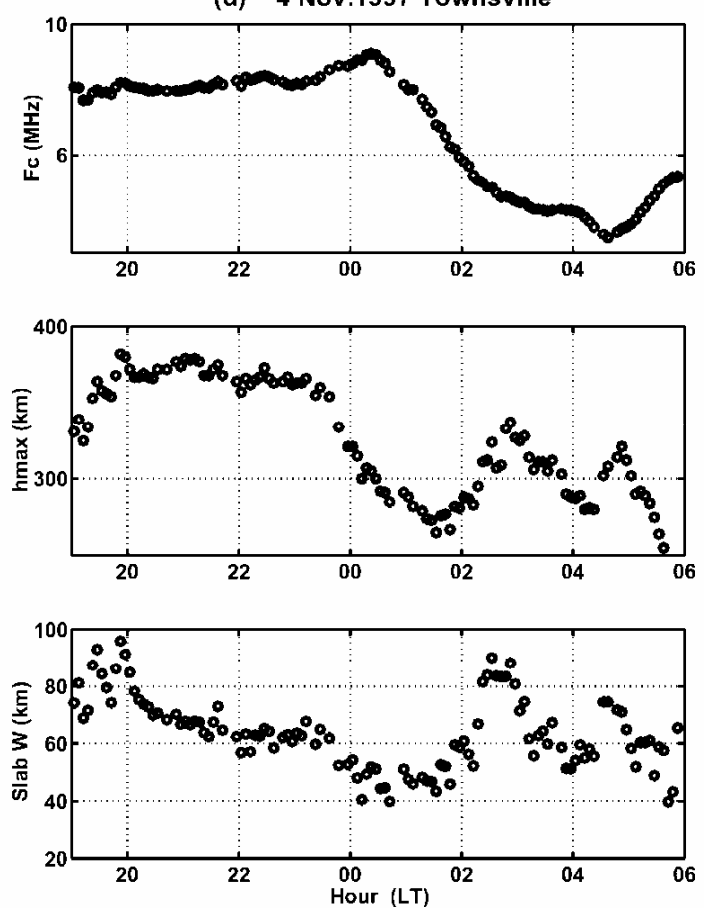

(b) 4 Nov.1997 Vanimo-Townsville
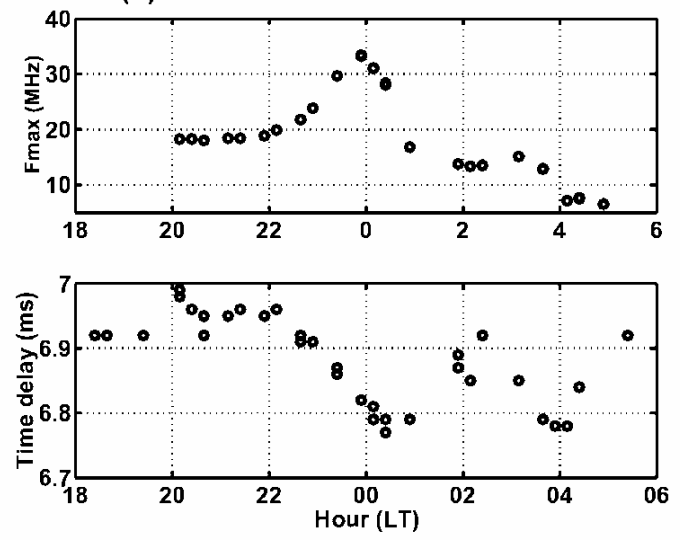

(c) 5 Nov.1997 Darwin
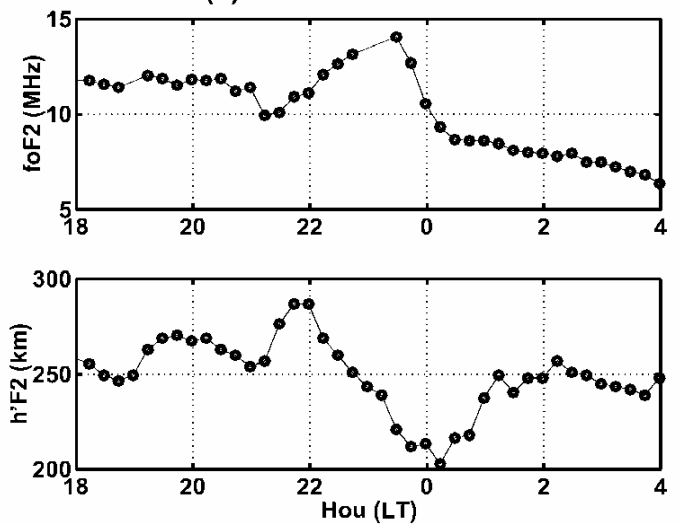

(e) 1 Nov.1997 Sumedang
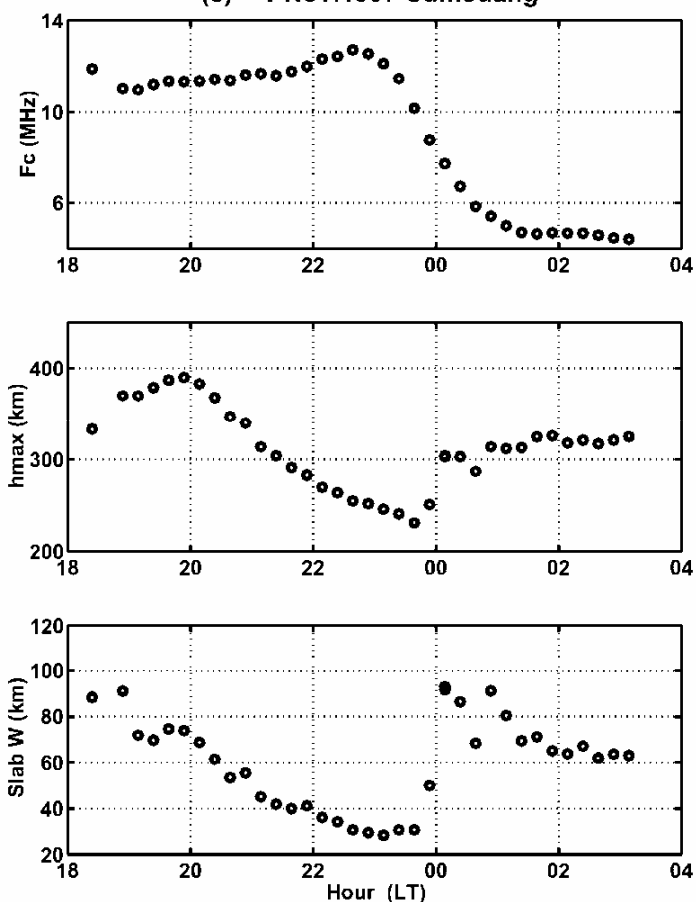

Fig. 5. Fc, $h m \mathrm{~F} 2$ and sub-peak slab width $W$ measured at Vanimo, Sumedang and Townsville. $F_{\max }$ and time delay observed over the oblique path Vanimo-Townsville and $f_{o} \mathrm{~F} 2$ and $\mathrm{h}^{\prime} \mathrm{F}$ observed at Darwin. 

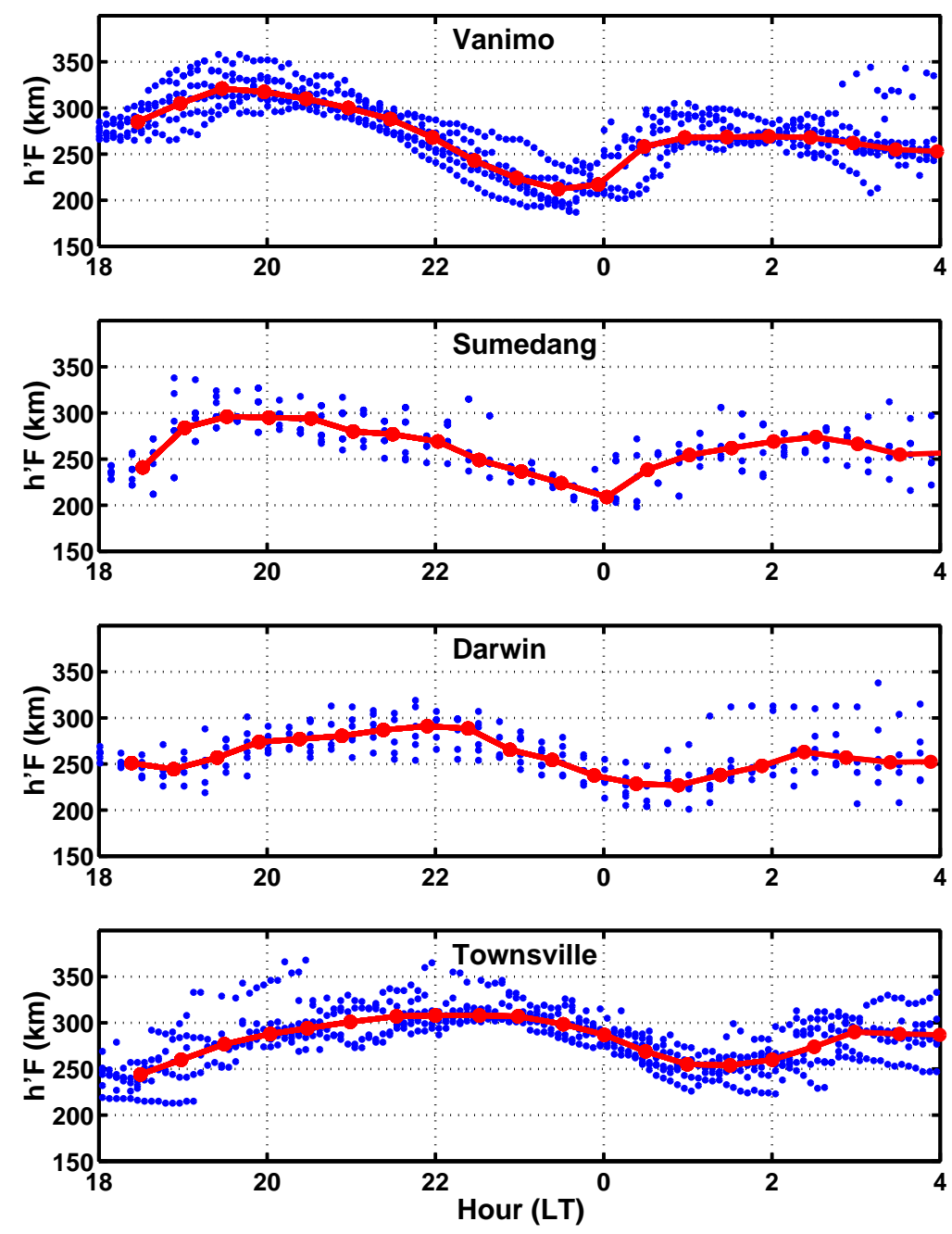

Fig. 6. Superimposed values of $h^{\prime} F$ (base ionospheric $F 2$ virtual height) as a function of time showing the delay in the post sunset fall in height with increasing distance from the equator as observed over a number of days. Median values are shown in red.

values were converted to equivalent virtual vertical velocity $(v)$ values using the usual formula

$v=-c . v /(2 . f)$

where $c$ is the speed of light, $v$ the observed Doppler frequency shift from the transmitted frequency and $f$ is the operating frequency. Plots of $f o \mathrm{~F} 2$ and virtual vertical velocity are given in Fig. 7 as measured on 1 November 1997. Vertical motion in this diagram is defined as being positive for upward velocities and downward for negative. The colour code for all vertical velocities may be read from the bottom panel of Fig. 7. Each vertical line in all three panels represents the contents of one ionogram.

The fall in peak layer height at Sumedang on the night of 1 November 1997 commenced at 20:00 LT and, as measured from Fig. 5 e, averaged $12 \mathrm{~m} / \mathrm{s}$ over a period of $3.5 \mathrm{~h}$. The downward drift then reversed to give an upward velocity of $39 \mathrm{~m} / \mathrm{s}$ averaged over the following $30 \mathrm{~min}$. These values are consistent with the velocities shown in Fig. 7 as calculated from the concurrent Doppler frequency shift measurements.

If the F2 layer moved uniformly as a whole, a constant velocity would be observed independent of frequency throughout the ionosonde sweep. However this rarely happens as is indicated in Fig. 7 by the spread in virtual velocity within each ionogram, seen most clearly in the bottom panel. The observed Doppler shift at each sounding frequency is an integrated function of ionisation change up to the reflection height (see discussion by Prabhakaran Nayar and Sreehari, 2004). In the present case, the variations in Doppler frequency and thus velocity appear to be dominated by changes in layer height. In Fig. 7, positive velocity values are associated with the sunset pre-reversal height rise and the recovery from the pre-midnight fall occurring after midnight. This pattern repeated on successive nights whenever the postsunset surge occurred. 

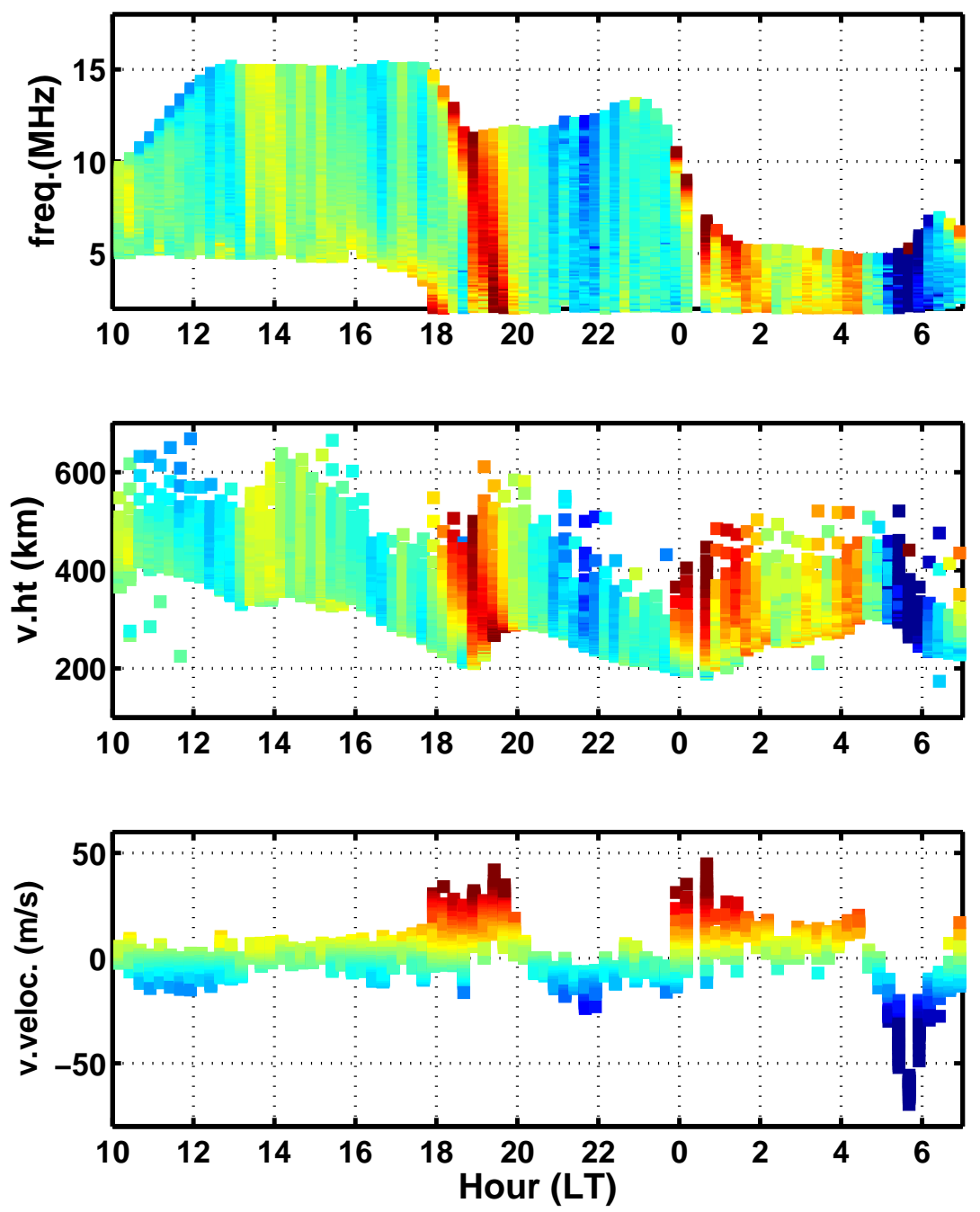

Fig. 7. Doppler derived vertical velocity plotted as a function of time (bottom), as a function of ionogram frequency (top) and virtual height (middle). The colour code for velocity used throughout can be read from the bottom panel in relation to the time dependence of velocity. Data from Sumedang as observed on the 1 November 1997.

In the upper panel of Fig. 7, the frequency at which the peak velocity occurs is seen to move down from the critical frequency to the lowest observed frequency during the period of post-sunset height rise between 18 to 20 LT. During the subsequent fall in height over the period 21-22 LT, the greatest downward (negative) velocity was present at the highest frequency (peak of the layer).

The lower panel of Fig. 7 shows a slight decrease in the downward velocity between 22-24 LT. This corresponds to a temporary reduction in the rate of fall of ionospheric base height at this time as seen in the middle panel of Fig. 7. The Doppler observations of Fig. 7 are consistent with those of the more limited Doppler measurement of Prabhakaran Nayar and Sreehari (2004) made on the magnetic equator at Trivandrum. The velocities observed during the post-sunset rise and subsequent fall and recovery in height were the highest values of velocity observed over the 24 -h period except for those associated with sunrise.

\section{Post-midnight events}

In recent years much theoretical work has gone into explaining the post-sunset height rise and the following reversal. Until recently it has been taken for granted that these changes are necessarily and uniquely associated with sunset conditions at the magnetic equator. However Fig. 5a provides an example of a Vanimo event commencing and finishing after midnight which is identical except in scale to the changes associated with sunset on this day. For this secondary event, the average velocity of the fall in peak height over a 1.4-h period from 01:30 LT was some $15.5 \mathrm{~m} / \mathrm{s}$ with a subsequent average rise velocity over $30 \mathrm{~min}$ of $64 \mathrm{~m} / \mathrm{s}$. These values 

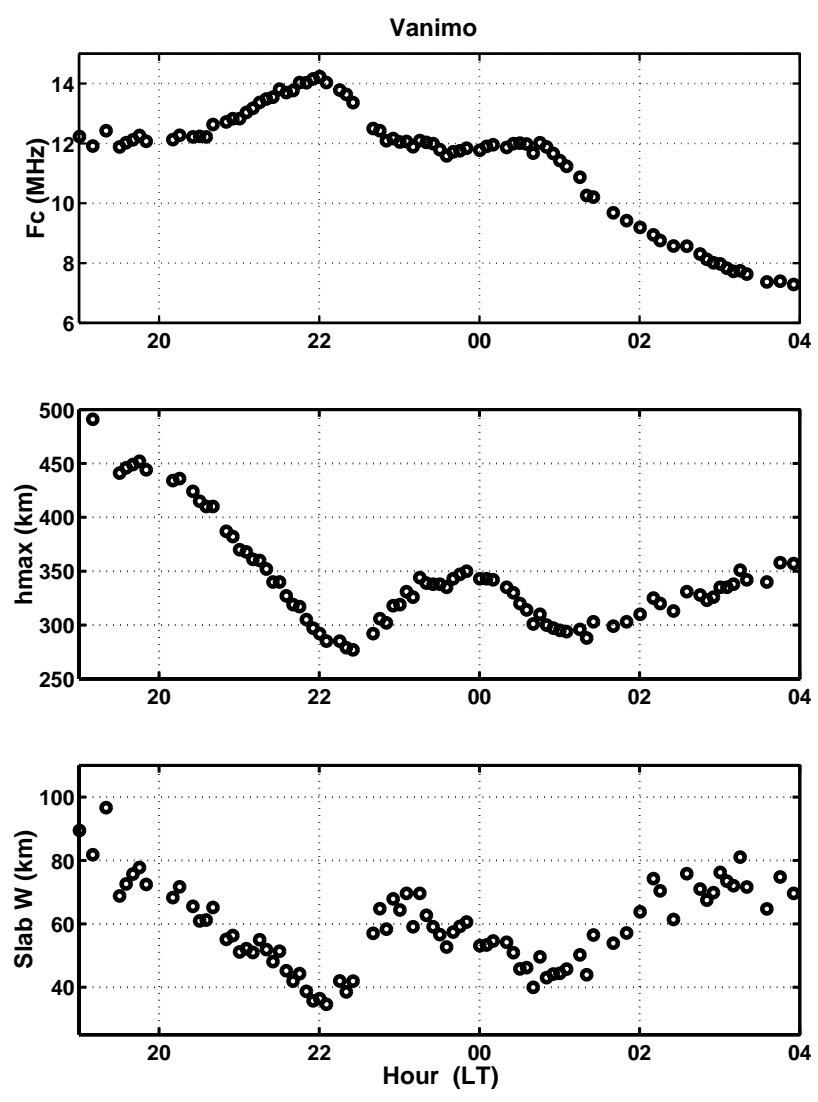

Fig. 8. Ionospheric critical frequency $F c$, peak height $h m \mathrm{~F} 2$ and sub-peak slab width $W$ observed at Vanimo on 24 February 1999 showing multiple similar events.

are essentially the same as those seen in the preceding sunset associated rise and fall. Although the increase in $f o \mathrm{~F} 2 \mathrm{ac}-$ companying the fall in ionospheric height and in ionospheric thickness is small in absolute terms with respect to the sunset event at Vanimo, it is still large in relative terms (about a 20\% increase in $f o \mathrm{~F} 2$ ) because of the lower values of electron density at this time. Apart from its lesser magnitude, this event is identical to the much stronger event associated with sunset which in turn represents the normally expected build-up and decay of the southern peak of the equatorial anomaly.

The post-midnight event on this night at Vanimo is also visible at Townsville (Fig. 5d) and, less clearly, on the path from Vanimo to Townsville (Fig. 5b). The time delay in the commencement of this event increased with latitude with the Townsville event occurring some $45 \mathrm{~min}$ after the event at Vanimo. The Vanimo-Townsville event occurred with an intermediate time delay consistent with its location. Such a strong correlation between all three measured parameters at all three sites over a distance of $2000 \mathrm{~km}$ in latitude would seem to require a major rise and fall in vertical ionisation drift at the southern peak of the equatorial anomaly with resultant delayed changes in distributed ionisation from the anomaly peak to its southernmost edge. Clearly such a possible correlation requires further investigation over a larger number of events.

Examination of a large number of Range-Time plots of $\mathrm{h}^{\prime} \mathrm{F}$ taken from low latitude ionosonde sites over a number of years indicate that such secondary phenomena are far from unusual. An example of true height data given by POLAN is shown in Fig. 8 as observed at Vanimo on the night of 24 February 1999. The post-sunset event follows the usual decrease in height, decrease in slabwidth, increase in $f o \mathrm{~F} 2$ and subsequent recovery over the period 20:00-24:00 LT, as described above. But this event is immediately followed by another which is identical in form though diminished in scale over the period 00:00-02:00 LT.

\section{Ionospheric storm effects 6-7 November 1997}

The patterns of $f o \mathrm{~F} 2$ and virtual height variation shown in Fig. 4 were consistent up to the night of 7 November. This was the night preceding a major low latitude negative ionospheric storm observed during daylight hours on the following day of 8 November. This storm was one of several discussed by Lynn et al. (2004). The negative storms there described are attributed to chemical changes produced by auroral up-welling reaching the equatorial region as driven by equatorward winds.

Observations of the sunset period over three days centred on the 7 November, 1997 at Vanimo are shown in Fig. 9. The normal post-sunset variations in $\mathrm{h}^{\prime} \mathrm{f}$ and $f o \mathrm{~F} 2$ occurred at Vanimo on the 6th and 8th. However no increase and subsequent decrease in post-sunset virtual height occurred on the evening of 7 November. Instead, following sunset, a slow rise in height occurred over a period of several hours during which the values of $f o \mathrm{~F} 2$ declined steadily with no sign of post-sunset surging. This may well be the diurnal variation when the night anomaly is absent and the usual zonal drift has stopped or reversed as a precursor to the negative ionospheric storm effect. On the following negative storm day, the day-time anomaly also failed to develop indicating a failure of the normal electrojet and fountain effect. However the ionosphere showed a partial recovery to its normal post-sunset variation on the following night.

\section{Discussion}

This paper shows the relationship between the four parameters $f o \mathrm{~F} 2, h m \mathrm{~F} 2$, sub-peak ionospheric slab thickness W and Doppler velocity during the build-up and decay of the night-time equatorial anomaly. Moreover the same relations in Fig. 6 are seen to be exhibited with diminishing intensity and increasing time delay as the latitude of the ionosonde increases from the night equatorial anomaly peak (Vanimo and Sumedang) to higher latitudes (Darwin and Townsville). The post-sunset surges in peak ionospheric critical frequency and the associated fall in ionospheric height continue to play 

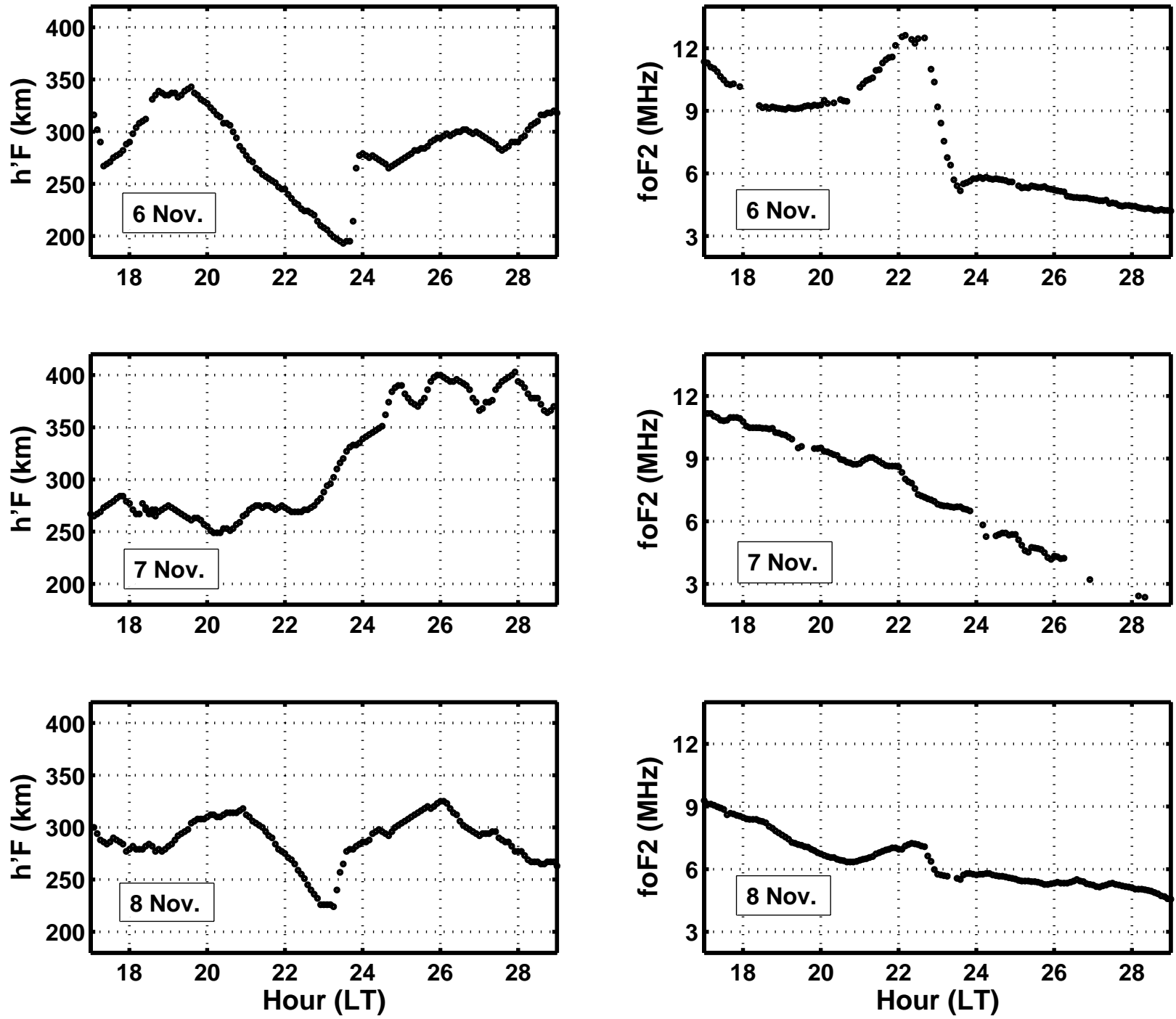

Fig. 9. Variations in base ionospheric height h'F and $f o \mathrm{~F} 2$ at Vanimo over the period 6-8 November 1997. Normal sunset vortex behaviour on the 6 November was stopped on the night of the 7 November preceding a negative ionospheric storm day. A partial recovery occurred on the following night.

their part in modifying the night ionosphere at and near the equinoxes as far south as Northern Australia. These latitudes are near the southern limits of the area dominated by a strong night-time equatorial anomaly.

The low-latitude vertical drift model of Scherliess and Fejer (1999) indicates that the post-sunset time variation in vertical drift velocity at the magnetic equator has little or no longitudinal dependence. Comparing their results with the observations of Fig. 3 shows that the fall in height at the lowest latitude site of Vanimo, commencing in the period 19:0020:00 LT, corresponds well with a change to downward drift at the magnetic equator at this time as given by Scherliess and Fejer (1999).
Arecibo in Puerto Rico is comparable to Townsville in terms of magnetic latitude and dip. Many papers from Arecibo refer to the "midnight collapse" of the ionosphere at this location. An example is given by Crary and Forbes (1986, their Fig. 2) in which the fall in peak height, the narrowing of the electron density profile and subsequent height rise is evident. The late occurrence of the fall in height is consistent with the time variation shown here for Townsville suggesting that there is a consistent delay with increasing latitude from the time the post-reversal fall in height occurs at the magnetic equator and that this delay is present at all longitudes. 
This paper shows post midnight events which mimic the height, fof 2 and ionospheric thickness variations associated with the sunset ionospheric vortex. The existence of this vortex now seems well-established. Kudeki and Bhattacharyya (1999) provide direct examples of the vortex drift in ionisation associated with sunset at Jicamarca. They also provide some evidence for the subsequent development of secondary vortices and discuss possible causes. In contrast, Nicolls et al. (2004) provide an example of a night at Arecibo for which large oscillations in F2 layer height were recorded. Each fall in height associated with the first two oscillations is accompanied by a surge in $f o \mathrm{~F} 2$ similar to that described here. The relationship of these oscillations with the normal "midnight collapse" is not directly commented upon. The second and larger rise and fall seems to have occurred at the expected time of such a collapse. Nicolls et al. (2004) attribute these oscillations to large-scale travelling ionospheric disturbances propagating equatorward from the auroral zone. However as noted in Sect. 7, the post midnight event seen on 4 November 1997 showed an increasing universal time delay with latitude and thus cannot be attributed to an equatorward TID unless it crossed the equator from the northern hemisphere..

Cécile et al. (1996) show an example of a large scale postmidnight variation in ionospheric height at an equatorial site in Africa. It may be that there are a number of possible sources of such events and that irrespective of the cause of a rise and fall in ionospheric height at these latitudes, the observed results can be the same in terms of the parameters discussed here.

The relationship between falling height and a temporary rise in $f o \mathrm{~F} 2$ is a common feature in day-time as well as at night and is probably a generic feature of large-scale TIDs. As pointed out by Nicolls et al. (2004) in relation to such TIDs, if the F2 layer falls (poleward wind), the peak density increases due to downward flux along dipping field lines and the layer also narrows due to recombination at the lower heights. The same result is produced by the sunset vortex although the latter is driven by electric fields. Difficulties of interpretation can thus arise when more than one phenomenon can produce a similar effect. In the light of these observations, more attention should be directed to the causation of large scale height variations in the post-midnight period at equatorial and low latitudes.

Some confusion also arises as to whether the pre-reversal height rise occurs after or before sunset depending on the definition of sunset used. If ground sunset is taken as the criterion, the pre-reversal height rise occurs mainly after sunset. However sunset at F2 peak heights occurs some hours later and at Vanimo often corresponds to the time of maximum height rise. In this respect, the pre- reversal height rise occurs before sunset at the peak height of the F2 layer at Vanimo.

The strong Equatorial Spread F (EQSF) associated with the rise of post-sunset bubbles produced by the RayleighTaylor instability were not obvious on any of the nights here described. Such bubbles typically develop at or shortly after the peak height of the post-sunset ionosphere is reached. Major EQSF usually associated with bubbles was observed to occur at Vanimo closer to the equinoxes than the nights examined here although the magnitude and duration of the preand post-reversal height variations were similar. This suggests that the occurrence of bubbles is more closely tied to the period in which the sunset terminator parallels the field lines. The occurrence of the pre-and post-reversal of sunset height variations seems to be less sensitive to this requirement.

This paper raises a number of questions for further experimental investigation at low latitudes and Southeast Asian longitudes. These include

1. whether the abrupt recovery of the electron density profile in slab thickness and maximum height following the post-sunset reversal is present at the magnetic equator as well as at the southern anomaly peak and beyond

2. the seasonal variation of the post-sunset height rise and fall and associated anomaly peak electron density

3. the sunspot cycle dependence.

4. the occurrence and ionospheric behaviour associated with major height rises and falls occurring later in the night

5. the relationship between ionosonde and satellite TEC measurements of the night-time equatorial anomaly

These matters and others are under current investigation. Theoretical questions remain relating to the latitudinal and time-dependence of the observations discussed here.

Acknowledgements. Topical Editor M. Pinnock thanks R. J. Stening for his help in evaluating this paper.

\section{References}

Abdu, M. A.: Outstanding problems in the equatorial ionospherethermosphere electrodynamics relevant to spread F, J. Atmos. S.P., 63, 869-884, 2001.

Balan, N. and Bailey, G. J.: Equatorial plasma fountain and its effects: Possibility of an additional layer, J. Geophys. Res. (Space Physics), 100, 21 421-21 432, 1995.

Cécile, J.-F., Vila, P., and Blanc, E.: HF radar observations of equatorial spread-F over West Africa , Ann. Geophys., 14, 411-418, 1996.

Crary, D. J. and Forbes, J. M.: The dynamic ionosphere over Arecibo: a theoretical investigation, J. Geophys. Res., 91(A1), 249-258, 1986.

Eccles, J. V.: Modeling investigation of the evening prereversal enhancement of the zonal electric field in the equatorial ionosphere, J. Geophys. Res., 103(A11), 26 709-26720, 1998.

Eccles, J. V., Maynard, N., and Wilson, G.: Study of the evening plasma drift vortex in the low-latitude ionosphere using San Marco electric field measurements, J. Geophys. Res., 104(A12), 28 133-28 144, 1999. 
Fejer, B. G.: Low latitude electrodynamic plasma drifts: a review, J. Atmos. S.-P., 53, 677-693, 1991.

Fejer, B. G., de Paula, E. R., Heelis, R. A., and Hanson, W. B.: Global equatorial ionospheric vertical drifts measured by the AEE satellite, J. Geophys. Res., 100(A4), 5769-5776, 1995.

Kudeki, E. and Bhattacharyya, S.: Postsunset vortex in equatorial F-region plasma drifts and implications for bottomside spread-F, J. Geophys. Res., 104(A12), 28 163-28 170, 1999.

Lynn, K. J. W., Harris, T. J., and Sjarifudin, M.: Combined TOPEX/Poseidon TEC and ionosonde observations of negative ionospheric storms seen at low latitudes, Ann. Geophys., 22, 2837-2847, 2004.

Nicolls, M. J., Kelley, M. C., Coster, A. J., González, J. J., and Makela, S. A.: Imaging the structure of a large-scale TID using ISR and TEC data, Geophys. Res. Lett., 31, L09812, doi:10.1029/2004GL019797, 2004.

Prabhakaran Nayar, S. R. and Sreehari, C. V.: Investigation of height gradient in vertical plasma drift at equatorial ionosphere using multifrequency HF Doppler radar, J. Geophys. Res., 109, A12308, doi:10.1029/2004JA010641, 2004.
Scherliess, L. and. Fejer, B.G.: Radar and satellite global equatorial $F$ region vertical drift model, J. Geophys. Res., 104(A4), 6829$6842,1999$.

Titheridge, J. E.: The real height analysis of ionograms: A generalized formulation, Radio Sci., 23, 831-849, 1988.

Tsunoda, R. T., Livingston, R. C., and Rino, C. L.: Evidence of a velocity shear in bulk plasma motion associated with the postsunset rise of the equatorial F-layer, Geophys. Res. Lett., 8, 807810, 1981.

Tsunoda, R. T.: Control of the seasonal and longitudinal occurrence of equatorial scintillations by the longitudinal gradient in integrated E region Pedersen conductivity, J. Geophys. Res., 90, 447-456, 1985.

Whalen, J. A.: Linear dependence of the post sunset equatorial anomaly electron density on solar flux and its relation to the maximum pre-reversal ExB drift velocity through its dependence on solar flux, J. Geophys. Res., 109, A07309, doi:10.1029/2004JA010528, 2004. 